\title{
Inflammatory fibroid polyp in ileum causing adult intussusceptions
}

\author{
SU Kafle ${ }^{1}$, P Upadhyaya $^{1}$, S Karki ${ }^{1}$, S Adhikary ${ }^{2}$, R Agarwal $^{2}$ \\ ${ }^{1}$ Department of Pathology and ${ }^{2}$ Department of Surgery \\ BPKIHS, Dharan, Nepal
}

\begin{abstract}
Inflammatory fibroid polyps are believed to occur in response to local noxious stimuli. They are uncommon benign but well documented solitary polypoid lesions occurring in gastrointestinal tract; most commonly in stomach. A rare entities of two adult intussusceptions due to inflammatory fibroid polyp in ileum has been reported in this article.
\end{abstract}

Keywords: intussusceptions, inflammatory fibroid polyp

\section{Introduction}

Inflammatory fibroid polyps are simple, localized, nonneoplastic lesions of gastrointestinal tract presenting most commonly as solitary sub mucosal sessile polypoid mass. ${ }^{1}$ Their most common site is stomach, followed by ileum and rarely the colon, duodenum and esophagus. These polyps are composed of fibrous connective tissue, blood vessels and an inflammatory cell infiltrate usually with many eosinophils. $^{2}$

Inflammatory fibroid polyps can be found in all age groups but peak incidence is between the sixth and seventh decades. ${ }^{3}$ Approximately $95 \%$ of all intussusceptions occur in children. ${ }^{4}$ The term "inflammatory fibroid polyp" was first proposed by Ranier and Helwig in 1953. ${ }^{5}$ Clinical symptoms may vary according to the location of the lesion. ${ }^{6}$ When it is in the stomach, most frequently seen symptoms are vomiting, epigastralgia and bleeding. Intussusceptions and obstruction are symptoms of the lesion located in the small bowel. On the other hand colicky pain, weight loss, diarrhea, bleeding and anemia are seen in colonic lesions. Although most of the cases are sporadic, a familial relationship has also been described. ${ }^{7}$

Address for correspondence

Dr Santosh Upadhyay Kafle

Assistant Professor, Department of Pathology, BPKIHS

Email:santosh735@hotmail.com
We report two-case of adult intussusceptions due to an inflammatory fibroid polyp (IFP) - a benign mesenchymal lesion of the gastrointestinal tract.

\section{Case Report}

First case: A 78 years male from Madhumalla, Morang presented with abdominal pain and distension. Further, he was admitted and operated for intussusceptions.

Gross features: Three intestinal bits were received in single container. The first intestinal bit measuring $21 \mathrm{~cm}$ in length and breadth varying from $3-4 \mathrm{~cm}$ had a polypoidal growth arising from mucosal surface measuring $4 \times 2.5 \times 2 \mathrm{~cm}$ and was $12 \mathrm{~cm}$ away from one of the resected end as shown in figure 1.

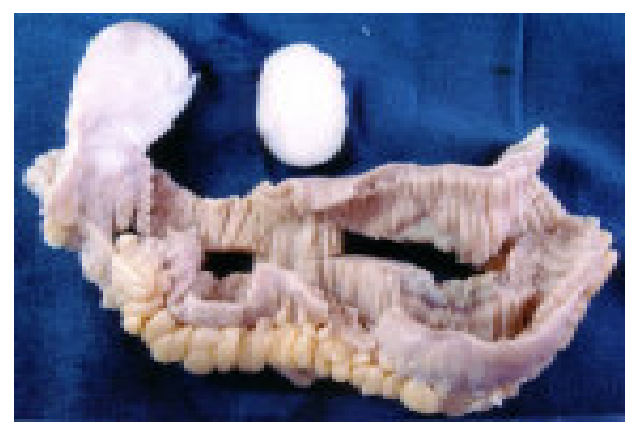

Fig.1: Gross specimen showing Intestinal mucosa with polypoidal growth 
Second case: A 60 years male from Dharan, Sunsari presented with pain abdomen and vomiting. Later on exploratory laparotomy was performed for intussusceptions.

Gross: Segment of small intestine measuring $23 \mathrm{~cm}$ in length and breadth varying from $3.5-4 \mathrm{~cm}$ was received. A sessile growth measuring $5 \times 3 \times 2.5 \mathrm{~cm}$ was identified arising from mucosal surface and was $2 \mathrm{~cm}$ away from one of the resected margin (proximal) as shown in figure 2 .

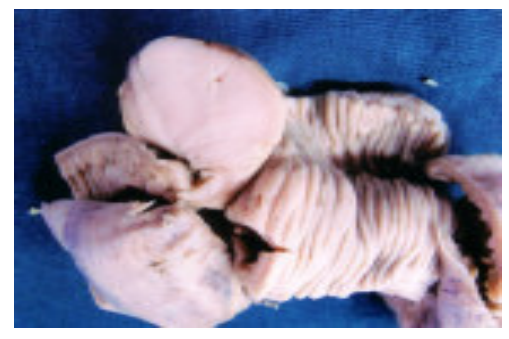

Fig.2: Gross specimen showing Intestinal mucosa with polypoidal growth

Cut surface of both growths showed gray white to gray brown solid smooth mass.

Microscopy of both the cases revealed mass of loose fibrous connective tissue arising from the submucosa and protruding into the lumen of the ileum. The lesion consisted of spindle shaped plump and stellate cells which were admixed with numerous blood vessels and having mixed inflammatory cells comprising of plasma cells, eosinophils and histiocytes (Fig.3 and 4). Perilesional tissue showed normal villi with increase in lymphocytes and plasma cells on microscopy. Bundles of smooth muscle cells separated by connective tissue stroma were also noted, which were highlighted by Masson Trichome stain on both the cases (Fig.5 and 6). Four (4) and two (2) lymphondes attached with the mesentery in case 1 and 2 respectively were reactive in nature.

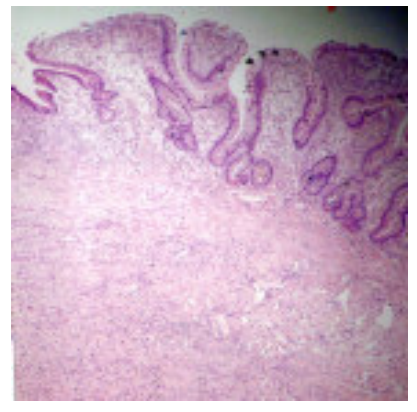

Fig.3: Microscopy of case 1 showing a lesion composed of spindle shaped cells admixed with chronic inflammation and many blood vessels (H\&E, x 400)

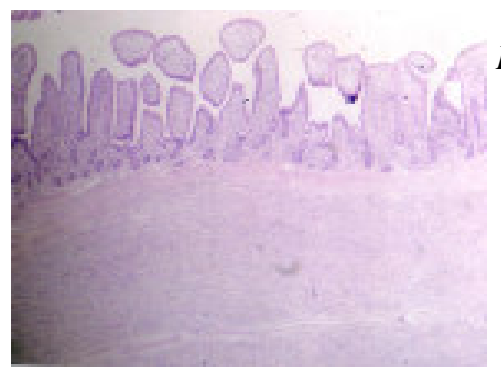

Fig.4: Microscopy of case 2 showing

lesion with oedematous and proliferative connective tissue

(H\&E, $x$ 400)

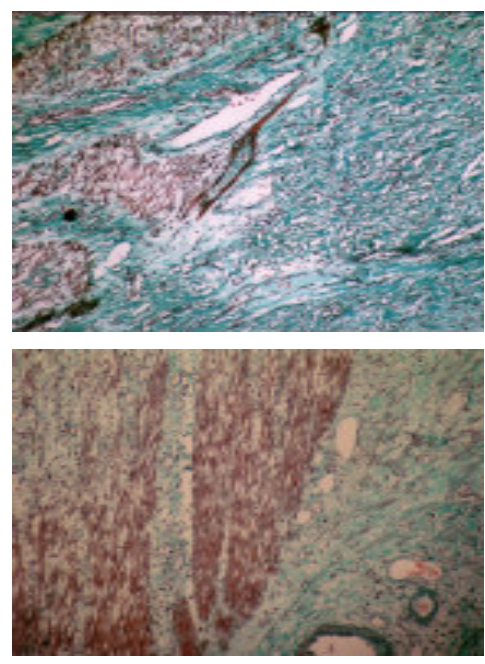

Fig:5: Microscopy showing special Masson Trichome stain (MT, x 100) of case 1

\section{Discussion}

IFPs are among the least common benign lesions of the gastrointestinal tract. They originate from the submucosa as a solitary or sessile lesion with an inflammatory basis. They can occur throughout the intestinal tract but most frequently in the gastric antrum and small bowel ${ }^{9}$. IFPs usually measure between 2 and $5 \mathrm{~cm}$ in diameter. However, there are also giant IFPs with a size of up to $12.5 \mathrm{~cm}$ in diameter having been reported ${ }^{10}$. IFP was first described by Vanek as a 'gastric submucosal granuloma with eosinophilic infiltration' in $1949^{11}$. Histologically, IFPs are characterized by vascular and fibroblast proliferation with an eosinophilic inflammatory response. The underlying cause of IFP remains uncertain. Many factors have been suggested as a trigger such as intestinal trauma or eosinophilic gastroenteritis.

IFPs are usually asymptomatic, identified during endoscopy or laparotomy. When they are symptomatic the clinical presentation is determined by the anatomic location. Gastric IFPs may lead to pyloric obstruction or anemia with chronic bleeding ${ }^{12}$. When they arise from the small bowel, intussusceptions is the most common clinical finding. 
Adult intussusceptions is a very rare condition, accounting for $1 \%$ of all adult bowel obstruction and occurs in only $5 \%$ to $16 \%$ of all intussuscepted cases $^{8}$. About $70 \%$ to $90 \%$ of intussusceptions cases are due to benign or malignant neoplasm as a lead point and IFPs, lipomas and adenomas are the benign causes of intussusceptions ${ }^{13}$ However, it has been shown that intussusceptions can occur without significant pathological cause ${ }^{14}$.

Unlike the more common idiopathic intussusceptions found in children, intussusceptions in adult patients still remains a surgical disease. The type of surgical procedure depends on the patient's medical history (previous operations, malignancy) and intra-operative findings ${ }^{15}$.

The optimal surgical management of intussusceptions in adult patients is influenced by two major factors: the presence of distinct malignancy and the local factors such as the degree of associated edema, and relative ischemia of the involved bowel. A wedge resection of affected bowel segment was performed in our case as very limited edema was observed at lead point of intussusceptions.

However, attempts at local removal of polyps through a limited enterotomy, or by wedge resection through edematous bowel, may be dangerous and healthy bowel margins must be secure during segmental resection.

\section{Summary}

Intussusception is a very rare cause of adult intestinal obstruction and IFP is one of the least common causes of this rare condition. Although IFPs are benign lesions, they can be treated adequately by resection of the involved bowel segments with macroscopically clear margins.

\section{References}

1. Johnstone JM, Morson BC. Inflammatory fibroid polyp of the gastrointestinal tract. Histopathology 1978; 2: 349-61.

2. Navan PJJ, Colina RF, Sanchez L, Cester CJ. Inflammatory fibroid polyp of the gastrointestinal tract. An Immunohistochemical \& electron microscopic study. Cancer 1983; 51: 1682-90.
3 De La Plaza R, Picarda A.L, Cuberes R, et al. Inflammatory fibroid polyps of the large intestine. Dig Dis Sci 1999; 44:1810-6.

4. Weilbaecher D, Bolin J, Hearn D, Ogden W. Intussusceptions in adults. Am J Surg. 1971. 121:531-535.

5. Helwig EB, Ranier A. Inflammatory fibroid polyps of the stomach. Surg Gynecol Obstet 1953; 96: 355-67.

6. Ali J, Qi W, Hanna SS, Huang S-N. Clinical presentations of gastrointestinal inflammatory fibroid polyps. CJS 1992;35: 194-8.

7. Anthony PP, Morris DS, Vowles KDJ. Multiple and recurrent inflammatory fibroid polyps in three generations of a Devon family: a new syndrome. Gut 1984; 25: 854-62.

8. Eisen LK, Cunningham JD, Aufses AH Jr: Intussusception in adults: institutional review. $J$ Am Coll Surg 1999, 188:390-395.

9. Wysocki AP, Taylor G, Windsor JA: Inflammatory fibroid polyps of the duodenum: a review of the literature. Dig Surg 2007, 24(3):162-168.

10. Harned RK, Buck JL, Shekitha KM: Inflammatory fibroid polyps of the gastrointestinal tract: Radiologic evaluation. Radiology 1992, 182:863-866.

11. Vanek J: Gastric submucosal granuloma with eosinophilic infiltration. Am J Pathol 1949, 25(3):397-411.

12. Gonul II, Erdem O, Ataoglu O: Inflammatory fibroid polyp of the ileum causing intussusception: a case report. Turk J Gastroenterol 2004, 15:5962.

13. Karamercan A, Kurukahvecioglu O, Yilmaz TU, Aygencel G, Aytaç B, Sare M: Adult ileal intussusceptions: an unusual emergency condition. Adv Ther 2006, 23:163-168.

14. Lvoff N, Breiman RS, Coakley FV, Lu Y, Warren RS: Distinguishing features of self-limiting adult small-bowel intussusception identified at CT. Radiology 2003, 227:68-72.

15. Yalamarthi S, Smith RC: Adult intussusception: case reports and review of literature. Postgrad Med J 2005, 81(953):174-177. 